EP-19

\title{
Surgical treatment of perihilar cholangiocarcinoma
}

\author{
Oleksii POPOV*, Oleksandr HRINENKO, Andrii ZHYLENKO, Oleksandr KORSHAK, \\ Andrii HUSIEV, Denis FEDOROV, Oleksandr OSTAPISHEN, Andrii KHOMIAK \\ Department of Liver Surgery and Transplantation, National Institute of Surgery and Transplantology, Ukraine
}

Introduction: One of the important and characteristic features of perihilar cholangiocarcinoma is tumor invasion to the area of the portal vein bifurcation, which occurs in $30 \%$ to $45 \%$ of cases. The aim of our study was to asses results of surgical treatment of perihilar cholangiocarcinoma with (Group 1) and without (Group 2) portal vein invasion.

Methods: From 2003 to January 2021 in the Department of Surgery and Liver Transplantation of the Ukrainian National Institute of Surgery and Transplantation, 170 patients with perihilar cholangiocarcinoma underwent major extended liver resections.

Results: We compared 79 (46.5\%) patients who received extended liver resection with portal vein resection with 91 (53.5\%) patients who underwent liver resections without vascular reconstructions. In the preoperative period, 150 (88.5\%) patients underwent decompression of the bile ducts, using percutaneous transhepatic cholangiostomy (PTBD) or retrograde endobiliary stenting. For patients with small remnant liver volume less than $40 \%$, in 42 cases we did preoperative PVE of a resected part of the liver. In 9 cases we made simultaneous PVE and PTBD. When choosing the volume of surgical intervention, we proceeded from the tumor type of Bismuth-Corlette classification, invasion into the portal vessels and the depth of the liver lesion. The portal vein reconstruction was in all cases performed in an "end-to-end". In all cases we made extended lymphadenectomy. Histologically, the tumor in 7 (11.5\%) cases was well differentiated, in 47 (77\%) cases-moderate differentiated, in 7 (11.5\%) cases-poor differentiated.

Conclusions: Aggressive surgical treatment for perihilar cholangiocarcinoma with portal vein resection can be performed. 